\title{
Innovation pédagogique en cours de français à l'université et son impact sur les compétences en langue écrite : un apprentissage différent?
}

\author{
ANNE ROHR \\ Université Toulouse 2 - Jean-Jaurès
}

\begin{abstract}
Résumé
Dans une formation à l'intention de futurs professeurs des écoles, nous avons comparé deux modes pédagogiques différents traitant d'un même contenu dans un enseignement de remise à niveau en français en Licence $1^{1}$. Nous cherchions à déterminer s’il existe un impact sur les apprentissages selon le mode innovant de la classe inversée et qui serait différent de celui obtenu avec un mode plus classique, non innovant. Les résultats de notre recherche font état de meilleures performances avec le mode inversé, ainsi que d'un profil d'erreur différent pour les deux groupes testés. Ces résultats sont interprétés dans le cadre de la théorie d'apprentissage de flexibilité représentationnelle ainsi que celui du changement conceptuel.
\end{abstract}

Mots clés

innovation pédagogique, apprentissage, enseignement universitaire, cours de français, effet classe inversée, types d'erreur

Introduction

Lorsque, en contexte universitaire, l'on s’intéresse aux compétences des étudiants francophones en langue écrite, s’impose alors rapidement le constat que les savoirs sont souvent mal maîtrisés. Ces connaissances lacunaires peuvent pénaliser les étudiants dans leur projet universitaire et professionnel. Les recherches spécifiques sur les facteurs déterminant l'échec ou la réussite en premier cycle de l'enseignement supérieur indiquent que les « lacunes dans la maîtrise de la langue » sont impliquées à hauteur de $25 \%$ dans l'échec en première année du premier cycle universitaire (Defays, Maréchal \& Mélon,2000 ; Romainville, 1997). Bellity, Gilles, l'Horty \& Sarfati (2016) montrent par ailleurs qu'un entraînement spécifique en français permet aux étudiants avec un niveau faible en français d'améliorer leurs résultats dans des disciplines tant littéraires que scientifiques.

Le domaine de l'orthographe grammaticale est particulièrement concerné. En effet, des productions, telles que « Le vocabulaire *sont les mots que *connaissent une personne ou qui appartiennent à la langue », «L'enseignante s'est *absenté » ou " Les livres ont été *donné » ou encore « La langue de l'enfant a *évoluer » ne sont pas rares et attestent de la difficulté que rencontrent les étudiants dans leur production écrite.

Ces difficultés pénalisent les étudiants dans leurs évaluations universitaires et lorsqu'il s'agit ensuite d'intégrer le monde professionnel, recruteurs / employeurs, collègues et clients critiquent, parfois sévèrement, ces défauts de connaissances en langue

\footnotetext{
${ }^{1}$ Le cursus de Licence correspond à celui du Baccalauréat canadien et Licence 1 (ou L1) fait référence à la première année de ce cursus.
} 
écrite. C’est notamment au moment du recrutement que les compétences manquantes portent préjudice aux candidats (Martin-Lacroux, 2016).

Plus préoccupant même, les enseignants du primaire, dont une des missions principales reste l'enseignement de la langue écrite, sont parfois peu sûrs de leurs savoirs ou produisent des erreurs qui peuvent enrayer la construction des bonnes représentations orthographiques chez les élèves.

Selon les résultats de Defays et al. (2000) et de Romainville (1997), ce problème est, au moins partiellement, à mettre en relation avec un constat plus global : en France, malgré le déploiement de divers outils et dispositifs, le taux d’échec et/ou de décrochage des étudiants en première année de licence reste très important. Selon les statistiques du Ministère de l'Enseignement Supérieur et de la Recherche, seuls 40,1\% de la cohorte des étudiants inscrits dans les universités françaises en L1 durant l'année universitaire 201415 passent directement en L2 à l’issue de leur L1 (MESR, 2016).

Cette situation constitue un enjeu majeur pour les universités qui multiplient depuis des années les initiatives afin d'augmenter les taux de réussite en L1.

Parmi les solutions envisagées, une des propositions fortes actuelles visant à améliorer l'implication des étudiants dans le processus d'apprentissage et dans la construction du savoir et savoir-faire est celle des pratiques pédagogiques innovantes, telles que la classe inversée, l'utilisation du numérique, et plus globalement, les pédagogies qui mettent les étudiants en activité (Freeman et al., 2014 ; Hamdan, McKnight, McKnight \& Arfstrom, 2013).

Le présent travail se situe dans le cadre des initiatives d'innovation pédagogique et propose, en conjuguant approche théorique et « expérimentation » pédagogique, au moyen d'une recherche-action (Lafontaine, 2016), d'examiner portée et effets de ces pratiques pédagogiques innovantes sur les capacités littéraciques des étudiants. Plus concrètement, dans une comparaison de plusieurs groupes d'étudiants ayant étudié un contenu littéracique identique via des approches pédagogiques différentes, nous examinons les taux de progression dans différentes tâches, ainsi que l'évolution des types d'erreurs ${ }^{2}$.

\section{Problématique et objectifs}

Dans le contexte d'un parcours d'études en sciences sociales et humaines comportant un volet destiné à de futurs professeurs des écoles, nous avons mis en place des enseignements de grammaire et d’orthographe du français en première année de licence afin d'améliorer les compétences littéraciques des étudiants.

L'une des interrogations soulevées par cet objectif, celle du dispositif pédagogique le plus pertinent et/ou efficient pour l'amélioration visée, forme le cadre de notre étude. Le choix et la mise en place de ce dispositif en tant qu' " ensemble cohérent constitué de ressources, de stratégies, de méthodes et d'acteurs interagissant dans un contexte donné pour atteindre un but » (Lebrun, Smidts \& Bricoult, 2011), qui, idéalement, agirait de manière efficace et durable sur les représentations cognitives des étudiants/apprenants,

\footnotetext{
${ }^{2}$ Les termes « profil d'erreurs », «typologie d'erreurs » et « type d'erreurs » sont définis comme suit : « Profil d'erreurs » renvoie à la configuration d'un ensemble d'erreurs de différents types qui sont dégagés par l'analyse systématique d'un corpus d'erreurs.

Une «typologie d'erreurs » signifie l'ensemble des types d'erreurs, mais non leur configuration particulière, i. e. le poids de tel ou tel type dans l'ensemble des données, et «type d'erreurs » renvoie à une seule catégorie de l’ensemble, définie par une propriété /caractéristique particulière.
} 
entraîne à son tour un ensemble de questions relatives au lien pédagogie ${ }^{3}$, (théories d')apprentissage et cognition (Eastes, 2013).

Notre démarche s'inscrit dans le champ des études visant une meilleure compréhension des processus cognitifs impliqués dans la construction des savoirs et savoir-faire en situation d'apprentissage universitaire.

Ces études ne peuvent pas (encore) se fonder sur un cadre théorique unifié, car elles convoquent différentes disciplines : la psychologie cognitive et ici plus particulièrement sa dimension psycholinguistique, la psychologie de l'apprentissage, ainsi que les sciences de l'éducation. Approches complémentaires, elles placent deux aspects au centre de notre démarche : (a) le processus d'apprentissage dans sa dimension cognitive mis en relation avec (b) le dispositif pédagogique déployé. Le choix de ce dispositif est alors d'un côté induit par la théorie des processus d'apprentissage et de l'autre côté par les théories d'apprentissage socioconstructivistes. Convoquer ces deux approches théoriques nous permettra d'établir un lien entre la progression des étudiants et certains processus cognitifs d'apprentissage ${ }^{4}$ qui sous-tendent cette progression dans telle ou telle situation pédagogique.

\section{Cadre conceptuel}

La démarche pédagogique de la classe inversée exporte à l’extérieur les activités habituellement réservées à la salle de classe et place celles que les élèves ou étudiants effectuent individuellement à la maison dans la salle de classe. C'est d'abord sous forme de documents écrits, capsules vidéos, etc. que les apprenants s'approprient individuellement et à leur rythme les contenus en dehors de la classe pour ensuite, durant le temps de classe, lors d'activités collaboratives entre pairs et d'échanges avec l'enseignant consolider l'acquisition de nouveaux savoirs et savoir-faire en situation d'application (Lebrun, 2017).

Considérée comme l'une des formes de pédagogie active, l'étudiant est mis en activité par l'appropriation personnelle des contenus, souvent via des dispositifs numériques, puis par l'expérimentation, le partage et l'élaboration de projets en classe. A ce titre, la classe inversée est généralement associée aux modèles d'apprentissage hybrides («blended learning ») (Lebrun, 2014).

Si ce cadrage très général fait consensus, le concept de classe inversée couvre cependant des réalités diverses et se décline en de multiples variations comme en attestent les enseignants pratiquant ce type de pédagogie à différents niveaux scolaires et dans différentes disciplines (cf. http://www.laclasseinversee.com/teacutemoignagesdenseignants.html). Ainsi, la partie externalisée peut consister, parmi d'autres, en la lecture de documents écrits, le visionnage de capsules vidéo, l’écoute de morceaux musicaux, la recherche de documents relatifs à un sujet donné, etc., activité(s) suivie(s) dans certains cas par un questionnaire à remplir ou une réponse à formuler à une question. Le travail en classe peut ensuite être très divers et prendre, par exemple la forme du montage d'un journal, ou de la gestion d'une ville virtuelle, ou encore de l'établissement de cartes mentales à partir des documents travaillés en dehors de la classe, etc.

En matière de théorie d'apprentissage, les théories du socioconstructivisme et du

\footnotetext{
${ }^{3}$ Nous utilisons le terme « pédagogie » dans le sens de méthodes et procédés d'enseignement.

${ }^{4}$ Par « processus cognitifs d'apprentissage », nous entendons la modification durable des représentations mentales et des schèmes d'action (Raynal \& Rieunier, 1997).
} 
socio-cognitivisme (Bruner, 2008 ; Piaget, 1997; Vygotsky, 1985) forment le socle conceptuel. L'apprentissage, en lien étroit avec l'interaction étudiants-étudiants et les échanges didactiques enseignant-étudiants, est envisagé comme le résultat d'activités sociocognitives qui permettent aux apprenants de construire des connaissances. Il s'agit autant d'acquérir de nouveaux savoirs et savoir-faire et de restructurer les connaissances préexistantes que de développer/approfondir les capacités d'apprendre, de comprendre, d'analyser et de maîtriser les outils dans des situations problèmes.

Rapprocher les théories socioconstructivistes et sociocognitives des recherches en psychologie cognitive de l'éducation semble alors incontournable lorsqu'on s'intéresse aux pédagogies innovantes, ici plus particulièrement à la méthode de la classe inversée. Des résultats de recherche attestent à la fois d'un meilleur apprentissage chez les étudiants en situation de métacognition à propos de leur propre processus d'apprentissage et d'une meilleure (re)structuration et mémorisation des contenus travaillés (Guemadji-Gbedemah, 2013).

Les résultats mesurant l'impact de la classe inversée sur les compétences et un meilleur apprentissage sont encore assez peu nombreux. Très majoritairement nordaméricaines, les études disponibles montrent globalement une amélioration des résultats chez les élèves et étudiants ayant bénéficié d'un dispositif inversé à condition que ce dernier ait été adapté au contenu et à la situation d'apprentissage (voir Hamdan et al., 2013 et Yarbro, Arfstrom, McKnight et McKnight, 2014 pour une revue). Toutefois, ces recherches portent essentiellement sur des expériences dans des disciplines comme les mathématiques, les sciences et technologies. Les sciences humaines et sociales ou les lettres et langues ne sont pas encore investies dans les mêmes proportions.

Par ailleurs, la classe inversée faisant partie des pédagogies actives, les effets qu'elle induit ne peuvent pas toujours être distingués de ceux d'un autre apprentissage actif.

Ainsi, Jensen, Kummer et Godoy (2015) publient une étude comparative entre classe inversée et travaux dirigés (désormais TD) avec pédagogie active non inversée. La recherche, conduite en contexte universitaire, concerne des enseignements en Sciences de la Vie et de la Terre. L'étude ne fait état d'aucune différence significative entre les deux groupes lors de l'examen de fin de semestre et rapporte une très bonne progression pour les deux groupes. L'interprétation de ce résultat va dans le sens d'un fort effet de l'apprentissage actif et des nombreuses interactions enseignant-étudiants qui ont bénéficié aux deux groupes. Selon cette étude, la classe inversée ne comporterait donc pas d'avantage repérable en comparaison avec d'autres formes pédagogiques actives. Dès lors que les étudiants se trouvent en situation d'apprentissage actif, appuyé par les échanges avec l'enseignant ainsi que par les interactions entre pairs, l'amélioration est tout à fait similaire.

Enfin, les méthodologies employées dans ces études peuvent varier fortement, utilisant par exemple des indicateurs comme les évaluations comparatives « avant-après », la variation générale des résultats pour l'ensemble d'une formation ou encore les taux de progression estimés par les étudiants eux-mêmes.

Le travail que nous présentons ici s’attache à évaluer quels sont les effets de la classe inversée sur l'apprentissage comparés à ceux d'un enseignement plus classique en cours de français. Ce cours est consacré à la reprise et la mise à niveau en grammaire et orthographe françaises en L1 dans un parcours préparant au master d'enseignement $1^{\mathrm{er}}$ degré.

Nous nous situons d'une part dans le contexte de la méta-analyse des effets des 
pédagogies actives établissant que le taux de réussite y est augmenté de 6\% (Freeman et al., 2014), puis des études montrant un impact globalement positif de la méthode inversée sur les performances des étudiants (Bishop \& Verleger, 2013). D’autre part, notre démarche est motivée par les travaux en neurosciences qui établissent un lien entre l'état actif des apprenants et l'apprentissage réussi (Dehaene, 2013) et nous formulons alors l'hypothèse suivante: les processus d'apprentissage et la transformation des représentations langagières travaillées en cours seront différents en fonction de la modalité pédagogique utilisée. Cette différence se traduira par des taux d'erreurs réduits en classe inversée ainsi que par des profils d'erreurs distincts dans les deux modes pédagogiques comparés.

L'amélioration spécifique que nous attendons porte sur l'application des règles d'accords verbaux, notamment celles des accords des divers participes passés.

La pratique pédagogique de la classe inversée apporterait un gain en termes de connaissances plus solidement ancrées et plus aisément récupérables dans différentes tâches impliquant la langue écrite.

\section{Méthodologie}

Notre démarche se décline en plusieurs étapes :

1. En premier lieu, nous avons fait un inventaire des erreurs d'orthographe grammaticale les plus fréquentes relevées dans des rédactions d'examen d'étudiants des années précédentes. Cet inventaire a conduit à repérer quelles étaient, pour les étudiants, les zones les plus problématiques dans la grammaire. En orthographe grammaticale, elles correspondent majoritairement aux phénomènes d'accords.

2. Lors de la deuxième étape, une séquence pédagogique ciblant les zones à problèmes identifiées préalablement a été conçue afin de répondre à ces besoins spécifiques de remise à niveau.

Chaque unité pédagogique était composée d’un Cours Magistral (désormais CM) dispensé à l'ensemble des étudiants, suivi de TD en plus petits groupes dont le contenu était décliné selon deux formes pédagogiques différentes : un enseignement « classique », non inversé qui se traduit par des exercices structuraux versus un enseignement sous forme de classe inversée dans laquelle les étudiants devaient conceptualiser et reformuler la règle, puis concevoir des questionnaires.

La séquence d'orthographe grammaticale portait sur les accords des participes passés. Lors du $\mathrm{CM}$, après un bref rappel des règles fondamentales régissant les accords des participes passés en français, les notions de «donneur » et de «receveur » d'accord étaient introduites. Ces dernières servaient de points d'ancrage pour la présentation des différents cas de figure des accords en lien avec des compléments d'objet directs et indirects ainsi que des verbes pronominaux.

Le CM était ponctué de petits quiz (effectués avec VotAR, système de vote en réalité augmentée, conçu par Poinsart en 2013), permettant aux étudiants de vérifier leur bonne compréhension du contenu traité précédemment. L’enseignant s’appuyait sur les résultats des quiz pour revenir sur les points du cours qui n'étaient pas suivis d'une majorité de bonnes réponses au quiz. 
La séance de TD suivant le CM pouvait prendre deux formes selon les groupes « non inversé » ou inversé.

Groupe non inversé :

- En début de TD, le groupe classe assistait à la projection de capsules vidéo, traitant des phénomènes d'accord. Nous avons choisi l'utilisation de matériel vidéo afin de contrôler le contenu strictement identique transmis aux étudiants des deux groupes étudiés sans une quelconque variation de la part de l'enseignant.

- Ensuite les étudiants réalisaient des exercices structuraux tels que :

- Correction de phrases avec erreurs d'accords

- Phrases à trous

- Transformation de phrases

- Choisir la bonne orthographe parmi différentes propositions

Parmi ces exercices, certains comportaient une consigne de justification.

- Chaque exercice était corrigé et commenté en groupe classe avant de passer à l'exercice suivant.

- $\quad$ Le TD, se terminait par un bilan - fait par l'enseignant - des règles et des exercices.

Ce type de situation pédagogique, sous-tendu par l'idée empiriste que l'apprentissage s'effectue via des processus d'imprégnation et de mémorisation, est donc largement frontal et transmissif. Il repose sur le principe behavioriste que le processus stimulus-réponse induit « conditionnement » et « renforcement » et que le « renforcement » s'opère à travers la répétition d’exercices se rapportant à des unités élémentaires de connaissances.

Groupe inversé :

- Avant le TD, les étudiants visionnaient les mêmes capsules vidéo que les étudiants du groupe non inversé pour des raisons, indiquées plus haut, de transmission identique du contenu.

La transmission de cette partie des connaissances reste par conséquent frontale - la modalité de présentation s’appuyant sur les technologies numériques constitue simplement une variation du mode que l'on pourrait qualifier de « frontal indirect » (Eastes, 2013).

La suite du TD s'organise en travail de groupe - chacun des groupes compte de 3 à 4 étudiants.

- À partir des capsules visionnées, chaque groupe vérifie que la compréhension de la règle est partagée par tous les pairs du groupe. Durant leurs échanges et afin d'étayer leurs arguments, les étudiants ont la possibilité de revisionner les capsules vidéo. C’est uniquement lorsque le groupe s'était assuré que tous ses participants partageaient les concepts et les règles travaillées, que l'étape suivante pouvait être entamée.

Ce stade du travail correspond globalement à l'enseignement par les pairs qui a 
pour objectif de repérer plus aisément - car effectué en petit groupe et loin du regard de l'enseignant - les conceptualisations incomplètes ou parfois erronées. Ce travail entre les pairs comporte par ailleurs l'avantage que les étudiants possédant déjà les concepts travaillés, identifient souvent mieux ou plus rapidement que ne le ferait l'enseignant où se situe un problème de compréhension ou d'interprétation chez un pair. Ils sont alors en mesure d'apporter des explications et des réponses appropriées.

Selon les résultats d'une méta-analyse des effets de l'enseignement par les pairs dans l'enseignement de la langue, Bowman-Perrot et al. (2013) relèvent un effet important pour l'apprentissage du vocabulaire et un effet moyen à important pour l'apprentissage de l'orthographe. Envisagé sous l'angle de la théorie du changement conceptuel, cette situation correspondrait au moment du conflit cognitif avec des conceptions préalables qui serait suivi d'une restructuration conceptuelle (Duit \& Treagast, 2003).

- L'étape suivante consistait en une reformulation des règles travaillées à destination d'un public d'apprenants. Ce travail collectif était consigné par écrit et rendu à l'enseignant qui pouvait ainsi s'assurer que l'ensemble des règles était correctement compris et reformulé.

L'objectif était ici de :

(a) rendre compte, à travers la trace écrite, de la représentation ${ }^{5}$ qu'ont les étudiants des règles travaillées,

(b) s'assurer d'un recouvrement sémantique adéquat entre les règles présentées dans les capsules vidéo et la reformulation,

(c) permettre une meilleure mémorisation des règles par l'activation, et éventuellement la restructuration des représentations conceptuelles associés aux règles.

- Pour finir, chaque groupe concevait une évaluation sous forme de questionnaire à choix multiples (QCM) à destination du public pour lequel il avait reformulé les règles. Ce dernier travail était également rendu à l'enseignant.

Le travail conceptuel et réflexif requis par cet exercice se situe à deux niveaux au moins :

a. Placer l'étudiant dans le rôle du concepteur d'évaluation par la formulation de questions pertinentes sur le contenu évalué induit une restructuration / réorganisation ainsi qu'un meilleur codage mnésique des connaissances.

b. Devoir concevoir des mauvaises réponses possibles pour le QCM nécessite d'identifier les éléments pouvant engendrer des erreurs, démarche sensée affiner les représentations correctes des règles et de leur application dans divers contextes.

3. Afin de mesurer l'évolution des compétences littéraciques des étudiants au fil des trois séances, deux types de tests leur étaient proposés à trois moments différents : une dictée et

${ }^{5}$ Par le terme représentation, nous entendons l'image mentale sur laquelle vont porter les opérations cognitives. 
un QCM avant le CM, entre le CM et les TD et après les TD.

\section{Les tâches}

Chaque dictée correspondait à un texte de 110 à 120 mots avec 10 à 11 participes passés reprenant les différents cas de figure des accords traités durant la séquence, par ex. «... ils se sont intéressés aux comédiennes qui les ont précédés ainsi qu’à celles qui leur ont succédé.... »

De même, contenant les différents types d'accord travaillés dans la séquence, chaque QCM était constitué de 10 questions avec 3 options de réponse, par ex. «Elles se les est (laver) enfin, les cheveux.
a) lavé
b) lavée
c)lavés»

La dictée précédait systématiquement le QCM afin que les propositions du QCM n’influencent pas les productions en dictée.

Le nombre d'étudiants par groupe se situait à $\mathrm{N}=50$ environ. Cependant, pour les analyses, seules ont été retenues les productions des étudiants présents à l'ensemble des trois tests, ce qui conduit à un nombre d'étudiants différents dans les deux groupes : N=44 dans le groupe non inversé et $\mathrm{N}=28$ dans le groupe inversé ${ }^{6}$.

\section{Résultats}

Compte tenu de notre problématique, la mesure d'analyse pertinente est celle du taux de progression ou taux d'évolution ${ }^{7}$ entre les trois moments de recueil de données. D’après nos hypothèses, nous devrions observer peu de différences entre les groupes inversé et non inversé après la première séance d'enseignement, car le CM a été suivi par l'ensemble des étudiants. En revanche, s’il existe un effet classe inversée, il devrait se révéler dans les mesures de progression après les séances de TD.

Les mesures des taux de progression exposées ici ont été effectuées sur les accords des verbes pronominaux pour :

(a) les deux types de situation pédagogique - inversé et non inversé - et

(b) les deux intervalles : avant-après CM et avant-après TD.

Nous présentons d'abord les taux d'évolution des réponses correctes dans les deux tâches, pour nous intéresser ensuite aux erreurs de surgénéralisation et aux erreurs d’accords non réalisés.

Tableau 1

Taux de progression en dictée des réponses correctes

\footnotetext{
${ }^{6}$ Le critère d'inclusion était la présence à tous les tests. Toute absence à l'un des tests, même le premier ou le deuxième, a conduit à ne pas retenir les autres productions de l'étudiant.

${ }^{7}$ Le taux d'évolution calcule l'évolution d’une grandeur numérique entre deux données. Il est donné en pourcentage, qu'il soit positif ou négatif. Si, par exemple, le nombre de bonnes réponses dans un groupe est de 30 à l'instant t1 et de 60 à l'instant t2, le taux de progression est de 100\%, et si l'on passe de 31 à 65 de bonnes réponses entre $t 1$ et $t 2$, le taux de progression est de 109\%.
} 




Tel qu’indiqué dans le tableau 1, en dictée, les taux de progression des réponses correctes d'application des règles d'accord des verbes pronominaux après la première séance (CM) montrent une différence de $9,6 \%$ avec une progression légèrement meilleure pour le groupe non inversé. Par la suite, la tendance s’inverse : après la seconde séance, celle avec les deux modes pédagogiques différents, le taux de progression est de plus de 15\% supérieur dans le groupe inversé. Lorsqu'on considère l'ensemble de l'évolution des réponses correctes en dictée, le mode inversé affiche de meilleurs résultats et accroit la progression des étudiants.

\section{Tableau 2.}

Taux de progression en QCM des réponses correctes



Contrairement aux résultats obtenus en dictée, dans la tâche de QCM, les taux de réponses correctes baissent après la première séance de cours $(\mathrm{CM})$, et ce pour les deux groupes, la baisse étant plus prononcée dans le groupe non inversé $(42,42 \%)$ que dans le 
groupe inversé (33,86\%). Ce résultat inattendu pourrait être imputable à un léger déséquilibre entre les 3 QCM constaté à posteriori : le QCM 2 (passé après le $\mathrm{CM}$ ) contenait plus de formes irrégulières $(\mathrm{N}=3)$ que le $\mathrm{QCM} 1(\mathrm{~N}=1)$. Une évolution positive des réponses correctes se dégage ensuite après la séance de TD dans les deux groupes. Tout comme pour la dictée, la hausse des performances est plus prononcée dans le groupe inversé $(23,26 \%)$ que dans le groupe non inversé (13,16\%) et confirme le constat qu'en comparaison au mode non inversé, le mode inversé induit une augmentation plus marquée des réponses correctes chez les étudiants.

Suite à cette première analyse générale des performances dans les deux tâches, nous avons considéré les types d'erreurs, plus précisément les erreurs de surgénéralisation (tableaux 3 et 4) et les erreurs de non-réalisation d'accords (tableaux 5 et 6).

Par erreurs de surgénéralisation, nous entendons l'application de la règle à des contextes non-appropriés, telles que "Ils se sont *succédés ». " Se succéder » étant un verbe introduisant un COI, il ne s'accorde pas - la forme correcte est donc «Ils se sont succédé ». Les commentaires des étudiants après le recueil des données attestent de l'application de la règle d'accord générale des verbes pronominaux, i.e. l'accord en genre et en nombre. Ils ont donc effectivement opéré une sur-extension de la règle à des situations où elle ne s'applique pas.

Tableau 3.

Taux de progression en dictée des erreurs de surgénéralisation

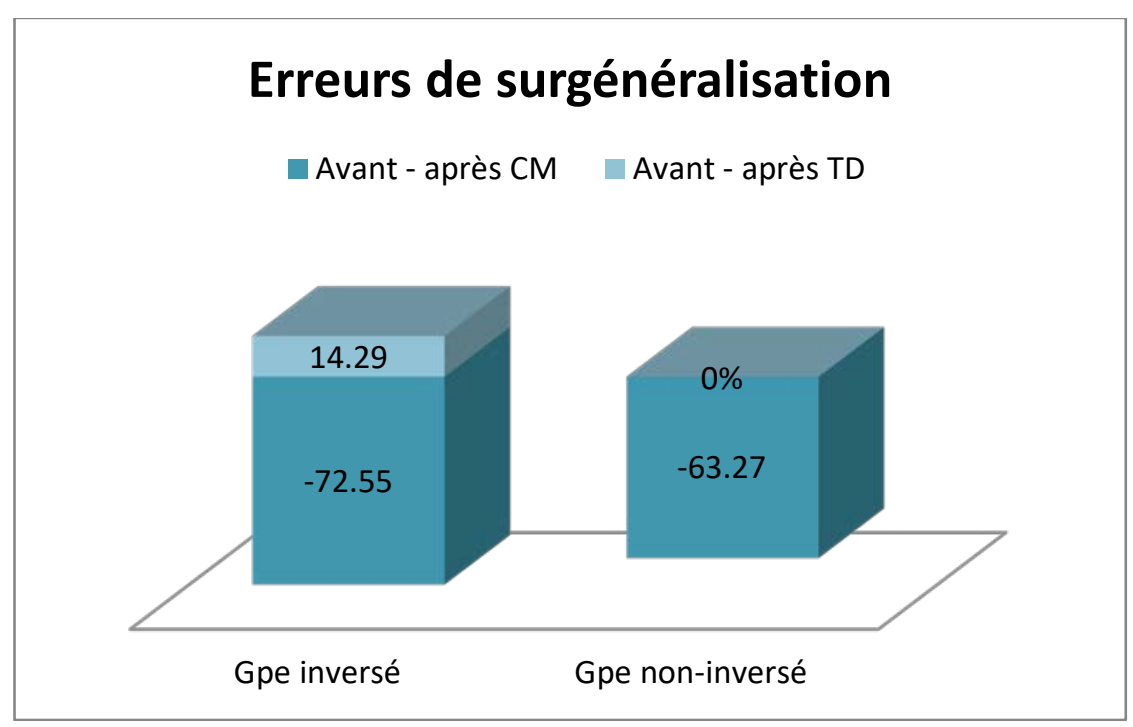

Les erreurs de surgénéralisation diminuent fortement dans les deux groupes après la première séance $(\mathrm{CM})$ avec respectivement un taux de progression de $-72,55 \%$ et $63,27 \%$ pour les groupes inversé et non inversé. Si dans le groupe non inversé, elles restent à un taux stable après la séance de TD, dans le groupe inversé, leur taux augmente de $14,29 \%$ par rapport au niveau constaté après $C M$. Résultat singulier à première vue, car une augmentation des taux d'erreurs n'est à priori pas attendue ici. Toutefois, replacé dans le contexte de la modification / l'évolution des représentations mentales durant 
l'apprentissage, ce résultat devient cohérent : lorsqu'un apprenant s'approprie une nouvelle règle, ou réajuste d'anciennes représentations erronées, il passe souvent par un stade où il étend l'application de la règle au-delà des contextes où elle s'applique réellement. Fayol et al. (1999) considèrent ce stade comme faisant partie du processus normal de l'apprentissage.

Si tel est le cas, les résultats dans la tâche de QCM devraient révéler la même tendance.

Tableau 4.

Taux de progression en QCM des erreurs de surgénéralisation

\section{Erreurs de surgénéralisation}

avant - après CM $\quad$ Avant - après TD

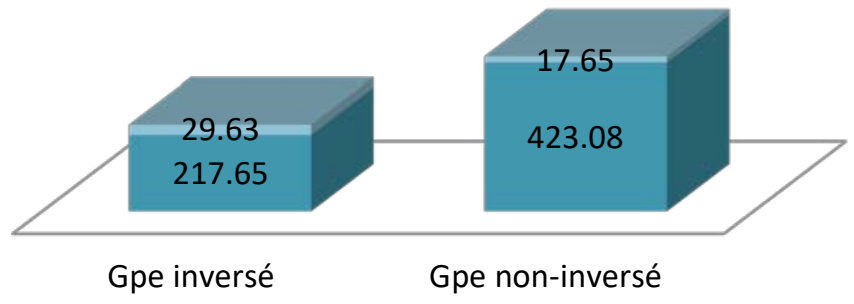

L’augmentation des erreurs de surgénéralisation, très importante au QCM après la séance de CM, est observée dans les deux groupes. Elle correspond à l'observation des taux d'évolution négatifs pour des réponses correctes discutée plus haut (cf. tableau2). Le fait que ce sont les erreurs de surgénéralisation qui augmentent fortement lors du deuxième relevé tend à confirmer notre hypothèse quant au déséquilibre des items irréguliers entre le QCM 1 et le QCM 2.

Quant à la comparaison des taux d'erreurs de surgénéralisation après la séance de $\mathrm{TD}$, elle révèle une progression plus forte dans le groupe inversé (29,63 \%) que dans le groupe non inversé (17,65\%). Ce résultat est par conséquent analogue à celui observé dans la tâche de dictée et peut s'inscrire dans notre cadre interprétatif : la systématisation de la règle peut engendrer sa sur-extension durant le processus d'apprentissage.

Afin de consolider notre lecture des résultats, nous avons analysé un autre type d'erreurs, les erreurs d'accords non-réalisés dans des contextes où leur réalisation était requise.

En effet, si le mode inversé conduit à une plus grande systématisation des règles d'accord que le mode non inversé, alors nous devrions observer des taux moins élevés dans le groupe inversé après les TD.

\section{Tableau 5.}

Taux de progression en dictée des accords non réalisés 


\section{Taux de progression des erreurs : accords non-réalisés}

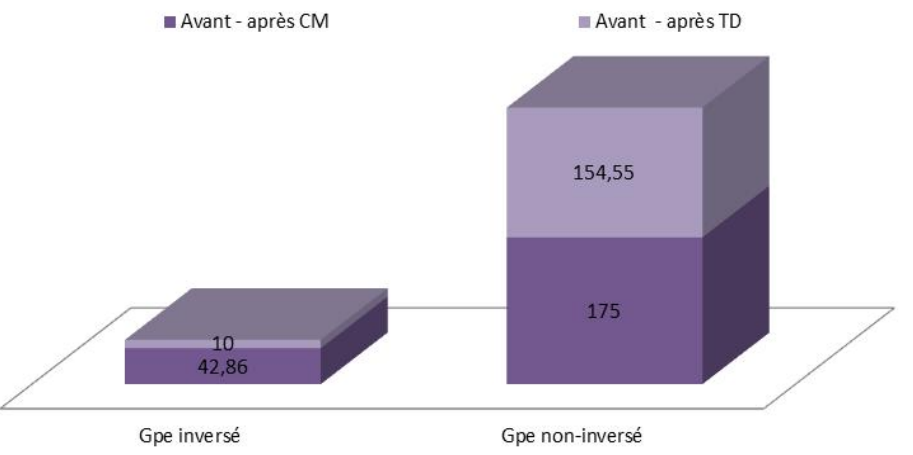

Le tableau 5 atteste en effet qu'en dictée, le groupe inversé réduit considérablement les erreurs d'accord non réalisé après la séance de TD (10\%) par rapport à ce même type d'erreurs après la séance de CM (42,86\%). Le groupe non inversé, en revanche, affiche un taux de progression important de ce type d'erreur après la séance de CM (175\%) et la réduction est moins importante après la séance de TD (154,55\%).

6.

La même analyse, conduite avec les données du QCM, est présentée dans le tableau

Tableau 6.

Taux de progression en QCM des accords non réalisés

\section{Erreurs d'accord requis non réalisé}



Tout en affichant des taux moins importants que dans la tâche de dictée, l'analyse du QCM met en évidence une tendance similaire : le taux de progression est respectivement de $63,71 \%$ et $43,17 \%$ pour les groupes inversé et non inversé après la séance de CM. Puis, le groupe inversé parvient à réduire le taux d'erreurs après la séance de TD $(-37,5 \%)$, alors que le groupe non inversé affiche des performances stables, sans évolution par rapport à la 
mesure avant la séance du TD (0\%).

Ces derniers résultats sont consistants avec l'interprétation que le mode inversé induit une systématisation plus ample dans l'application de la règle tandis que le groupe non inversé ne parviendra pas à ce stade de l'apprentissage après le même nombre d'heures d'enseignement.

\section{Discussion}

Les résultats qui viennent d'être exposés apportent plusieurs éléments de réponse aux questions que nous nous sommes posées. Dans une situation de comparaison directe entre un mode pédagogique inversé et un mode non inversé, la progression générale calculée en taux de réponses correctes en fin de séquence - est meilleure en mode inversé. Ce résultat est complété par une analyse du profil d'erreurs qui diffère selon le mode pédagogique.

À l'instar des recherches sur les effets des pédagogies actives (Freeman et al., 2014), et parmi elles, la classe inversée (Hamdan et al., 2013 ; Yarbro et al., 2014), nos données révèlent une amélioration des performances, ici dans un contexte d'apprentissage littéracique. La progression constatée est même plus importante (respectivement 18,33\% en dictée et 23,26\% en QCM) que la moyenne de 6\% rapportée par Freeman et al. (2014) dans leur méta-analyse des effets des pédagogies actives. En outre, l'effet semble indépendant des modalités de compréhension ou de production puisqu'il ressort dans les deux tâches : dictée et QCM. Non trivial, ce point laisse à penser que les représentations construites concernent tout à la fois la production et la compréhension écrite. En d'autres termes, les représentations en jeu ici seraient centrales, de type amodal ${ }^{8}$, et desserviraient les entités/modules cognitifs plus périphériques requis dans le traitement des tâches de dictée et de QCM.

Replacés dans le contexte de théories d'apprentissage, nos résultats s'accordent avec la théorie du changement conceptuel issue des modèles socioconstructivistes et le concept de conflit cognitif. Le changement conceptuel désigne des voies d'apprentissage empruntées par les étudiants partant des conceptions avant instruction aux concepts à apprendre (Duit \& Teagust, 2003). Ce changement est favorisé par la situation de conflit (socio-)cognitif que comporte le mode inversé, dans le sens où la confrontation avec les représentations conceptuelles des pairs conduit à une restructuration du savoir. Dans le mode pédagogique de la classe inversée, les étudiants se trouvent en situation de métacognition qui est vue comme le médiateur du changement conceptuel (Georghiades, 2000).

Si l'on considère ensuite les types d'erreur les plus fréquents produits par les étudiants, deux d'entre eux se démarquent particulièrement: les erreurs de surgénéralisaiton et les erreurs de non réalisation de l’accord.

Les taux d'évolution révèlent que le groupe inversé est plus sujet à la surgénéralisation après la phase 2 (TD) dans les deux tâches.

Ces erreurs de surgénéralisation ne sont pas de simples erreurs de performances dues à un défaut d'attention ou une connaissance insuffisante du fonctionnement de la langue - elles obéissent plutôt à une « logique de l'erreur » (Fayol, Thevenin, Jarousse et Totereau, 1999). Le processus de surgénéralisation est bien connu dans le cadre de

${ }^{8}$ Représentations conceptuelles abstraites indépendantes des modalités particulières liées à la production ou à la compréhension du langage. 
l'acquisition du langage ainsi que dans l'apprentissage de la langue écrite où on l'attribue au fait que l'enfant applique une procédure déjà connue à une situation nouvelle. Il serait alors le résultat du fonctionnement cognitif normal consistant en des processus d'actions stables. Nos données indiquent que ce processus de surgénéralisation est toujours à l'œuvre chez adulte, dès lors qu'il se trouve en situation d'apprentissage de production écrite. La différence entre les groupes inversé et non inversé ajoute un éclairage supplémentaire : selon le mode pédagogique utilisé, il semble possible d'influer sur la systématisation de l'utilisation d'une règle. Et contrairement à ce qui serait attendu dans le cadre de la théorie behavioriste, ce n'est pas la répétition de l'application de la règle via des exercices structuraux (mode non inversé) qui systématise le mieux les apprentissages. Nos résultats témoignent, au contraire, d'une systématisation plus importante avec le mode inversé, celui qui conduit les apprenants via une posture réflexive et métacognitive à un meilleur ancrage de la règle travaillée. Cette interprétation est tout à fait compatible avec le schéma classique de l'apprentissage en « $\mathrm{U}$ » dans l'approche théorique de la flexibilité représentationnelle (Karmiloff-Smith, 1992), où, après une première phase de l'apprentissage correspondant à la mémorisation des formes rencontrées, la deuxième phase consiste en la mise en relation des formes, l'identification des similarités entre formes et à l'extraction de règles. C'est lors de cette phase que surviennent les erreurs de surgénéralisation. Elle est suivie de la dernière phase durant laquelle l'ensemble des bonnes formes est appris. En raison du renforcement en mémoire de la liste des exceptions, voire d'une autre règle pour des cas moins fréquents, la règle déclenchant la surgénéralisation serait bloquée, ce qui aboutirait à une production conforme à la norme de la langue (Pinker, 2001).

A l'opposé, les erreurs de non réalisation d'accord aux endroits où l'accord est requis apparaissent majoritairement dans le groupe non inversé. Le mode classique, non inversé, avec la pratique d'exercices structurels suivie de corrections en groupe classe n'aboutit pas au même degré de systématisation dans l'application de la règle travaillée. Cette pratique, qui trouve son fondement théorique dans le behaviorisme (Bloom, Engelhart, Hill, Furst \& Krathwoh, 1956), soutient que le «conditionnement» et « renforcement » à travers la répétition d'exercices est la procédure indiquée lorsqu'il s'agit d'ancrer la trace des nouvelles connaissances en mémoire et conduit à l'utilisation systématique et correcte de la règle. Au contraire, nos résultats témoignent du fait que la meilleure assimilation est obtenue avec une démarche réflexive, quand bien même cette démarche fait passer par la phase basse du ' $U$ ' de Karmiloff-Smith (1992), celle de l'extraction de règles (Pinker, 2001) que les apprenants « surexploitent » pendant un temps, avant d'affiner leurs représentations et d'y intégrer les cas de figure moins fréquents et non solubles par la règle générale.

Considérés conjointement avec les surgénéralisations, l'évolution des taux d'erreurs de non-réalisation d'accord est compatible avec notre interprétation, par exemple, l'application de la procédure la plus fréquente et régulière. Dans le cas des accords requis, cela se traduit logiquement par une diminution des taux d'erreurs dans le groupe inversé après la séance de TD.

Pourrait-on alors envisager un apprentissage différent en termes de processus cognitifs selon le mode pédagogique utilisé ? Il serait prématuré de conclure à une telle différence, car il est possible que le groupe non inversé parvienne un peu plus tard à la même étape de surgénéralisation que le groupe inversé. L'interrogation qui émerge de nos observations concerne plutôt les raisons qui sous-tendent cette différence : s'agit-il de 
l'aspect multidimensionnel des tâches proposées en mode inversé (vérification de la compréhension des pairs, reformulation des règles, conception d'une évaluation) qui coderait des traces plurielles, plus robustes en mémoire ? Et ces traces permettraient de récupérer l'information plus rapidement, et peut-être via différentes voies d'accès à l'information?

Elargir le genre de comparaison que nous avons mené à un semestre entier et à des contenus divers consoliderait nos données et fournirait des éléments de réponse à ces nouvelles questions.

\section{Conclusion}

À l'instar des études sur les effets de la pédagogie inversée, majoritairement effectuées dans le contexte d'enseignements scientifiques (Freeman et al., 2014, Guedmadji-Gbedemah, 2013 ; Hamdan et al., 2013 ), nous constatons l'impact du mode classe inversée sur les résultats des étudiants dans un enseignement visant une amélioration des compétences littéraciques en français. Notre comparaison a mis à jour deux effets du mode inversé sur les performances des étudiants : de meilleurs résultats que le mode dit " classique », non inversé, ce qui concorde notamment avec les méta-analyses (BowmanPerrot et al., 2013 ; Hamdan et al., 2013). Dans la mesure où la classe inversée fait partie des pédagogies qui mettent les étudiants en activité, suscitent une posture réflexive et métacognitive, nos analyses corroborent les études, encore trop peu nombreuses, sur l'impact de la pédagogie inversée.

C'est toutefois le second résultat qui met à jour un effet particulier et nouveau : tout en améliorant les performances générales des étudiants ayant suivi l'enseignement inversé, le type d'erreur 'surgénéralisation' est plus fréquent dans ce groupe en comparaison avec celui qui a suivi un enseignement non inversé. Ces erreurs de surgénéralisation augmentent même après la séance inversée alors que les erreurs d'accords non-réalisés diminuent. La mise en relation de nos résultats avec la « logique de l'erreur » (Fayol et al, 1999 ; Pinker, 2001), l'évolution des représentations durant le processus d'apprentissage (KarmiloffSmith, 1992) et les propositions émergeantes du domaine de la neuro-éducation ou neuropédagogie (Dehaene, 2008 \& 2013) oriente vers une nouvelle perspective de recherche.

La question qui se pose n'est alors plus celle de la seule efficacité de tel ou tel mode pédagogique, mais celle de l'éventuelle différence dans la modification de représentations mentales générée par tel ou tel mode pédagogique. Nous aurons alors besoin de combiner les concepts venant de la linguistique et qui sont utilisés en cours de français avec la théorie du changement conceptuel ainsi que le cadre théorique employé en neurosciences, plus précisément en neuro-pédagogie.

La limite de notre étude est sa courte durée, à savoir quatre semaines entre le premier et le dernier relevé. Afin de consolider nos résultats, il sera nécessaire de suivre l'évolution de cette modification sous forme d'étude longitudinale. Ce n'est qu'à cette condition que l'on pourra évaluer plus précisément la portée durable en termes de changement conceptuel du mode inversé.

\section{Références}

Bellity, E., Gilles, F., L’Horty, Y., \& Sarfati, L. (2016). Faut-il encourager les étudiants à améliorer leur orthographe ? Travail Emploi et Politiques Publiques - Rapport de recherche, 2016-1, 1-41. 
Bishop, J., \& Verleger, M. (2013). The flipped classroom: A survey of the research. 120th ASSE Annual Conference \& Exposition. Repéré à http://www.asee.org/public/conferences/20/papers/6219/view

Bloom, B., Engelhart, M., Hill, M., Furst, E., \& Krathwoh, L.D. (1956). Taxonomy of educational objectives. Handbook, cognitive domain. New York, NY : Longman.

Bowman-Perrott, L., Davis, H., Vannest, K., Williams, L., Greenwood, C., \& Parker, R. (2013). Academic benefits of peer-tutoring : a meta-analytic review of single-case research. School Psychology Review, 42(1), 39-55.

Bruner, J. (2008). L'éducation, entrée dans la culture. Paris : Retz.

Defays, J. M., Maréchal, M., \& Mélon, S. (2000). La maîtrise du français : du niveau secondaire au niveau supérieur. Bruxelles : De Boeck Université.

Dehaene, S. (2008). Cerebral constraints in reading and arithmetic: Education as a “neuronal recycling”' process, in : A. M. Battro, K. W. Fischer \& P. J. Léna (Eds.), The educated brain: Essays in neuroeducation (pp. 232-247). Cambridge: Cambridge University Press.

Dehaene, S. (2013). Les quatre piliers de l'apprentissage, ou ce que nous disent les neurosciences. Repéré http://www.paristechreview.com/2013/11/07/apprentissageneurosciences/\#.Un7NhLLg228

Duit, R., \& Treagust, D. F. (2003). Conceptual change : a powerful framework for improving science teaching and learning. International Journal of Science Education, 25(6), 671-688.

Eastes, R.-E. (2013). Processus d'apprentissage, savoirs complexes et traitement de l'information : un modèle théorique à l'usage des praticiens, entre sciences cognitives, didactique et philosophie des sciences. Thèse de doctorat, Université Paris 1. Repéré à http://www.theses.fr/2013PA010593.

Fayol, M., Thévenin, M.G., Jarousse, J.-P., \& Totereau, C. (1999). From learning to teaching to learning french written morphology, in : T. Nunes (Eds.), Learning to read: An integrated view from research and practice (pp. 43-64). Dordrecht : Kluwer.

Freeman, S., Eddy, S. L., McDonough, M., Smith, M. K., Okoroafor, N., Jordt, H., \& Wenderoth, M. P. (2014). Active learning increases student performance in science, engineering, and mathematics. PNAS, 111(23), 8410-8415.

Georghiades, P. (2000). Beyond conceptual change learning in science education: focusing on transfer, durability and metacognition. Educational Research, 41(2), 119-139.

Guemadji-Gbedemah, T. E. (2013). Neurosciences et pédagogie. Thot Cursus formation et culture numérique. Repéré à http://cursus.edu/article/20086/neurosciences-pedagogie/\#.VTUeiJObLIW.

Hamdan, N., McKnight, P., McKnihgt, C., \& Arfstrom, K. (2013). A review of Flipped Learning.Repéré à http://flippedlearning.org/wpcontent/uploads/2016/07/LitReview_FlippedLearning.pdf

Jensen, J. L., Kummer, T. A., \& Godoy P. D. d. M. (2015). Improvements from a flipped Classroom may simply be the fruits of active learning. CBE - Life Sciences Education, 14, 1-12.

Karmiloff-Smith, A. (1992). Beyond Modularity : A Developmental Perspective on 
Cognitive Science. Cambridge : MIT Press.

Lafontaine, L. (2016). Recherche-action-formation au préscolaire et au 1er cycle du iscepprimaire : pistes méthodologiques, in : I. Carignan, M.-C. Beaudry \& F. Larose (Eds.), La recherche-action et la recherche-développement au service de la littératie (pp. 36-62). Sherbrooke : Les Éditions de l'Université de Sherbrooke.

Lebrun, M., Smidts, D., \& Bricoult, G. (2011). Comment construire un dispositif de formation ? Paris : De Boeck Supérieur.

Lebrun, M. (2014). Essai de modélisation et de systémisation du concept de Classes inversées. Blog de Marcel. Repéré à http://bit.ly/ML-Classes-inversées.

Lebrun, M. (2017). Les classes inversées, variétés et effets différenciés. Cahiers pédagogiques, 537. Repéré à http://www.cahiers-pedagogiques.com/Les-classesinversees-variete-et-effets-differencies.

Martin Lacroux, C. (2016). L’évaluation des compétences orthographiques lors du processus de recrutement une analyse expérimentale des décisions et des discours d'un échantillon de recruteurs. La revue des sciences de la gestion, 277, 79-89.

Ministère de l'Enseignement Supérieur et de la Recherche (2016). Note flash du 23.11.2016. Parcours et réussite aux diplômes universitaires : les indicateurs de la session 2015. Repéré à http://www.enseignementsuprecherche.gouv.fr/pid25366/acces-thematique.html? theme $=263 \&$ subtheme $=280$

Piaget, J. (1997). Commentaire sur les remarques critiques de Vygotski concernant le langage et la pensée chez l'enfant et le jugement et le raisonnement chez l'enfant. In : Vygotski, L.S. (1997). Pensée et Langage. (pp. 387-399). Paris : La Dispute. Repéré à http://www.fondationjeanpiaget.ch/fjp/site/textes/VE/JP62_Vygotski(francais).pdf

Pinker, S. (2001). Four decades of rules and associations, or whatever happened to the past tense debate ?, in : Dupoux, E. (Eds.) Language, brain and cognitive development: Essays in honour of Jacques Mehler (pp. 157-179). Cambridge : MIT Press.

Poinsart, S. (2013). VotAR. Système de Vote en réalité augmenté. Repéré à https://votar.libre-innovation.org/index.fr.html

Raynal, F. \& Rieunier, A. (1997). Pédagogie, dictionnaire des concepts clés. Apprentissage, formation, psychologie cognitive. Paris, France : ESF.

Romainville, M. (1997). Peut-on prédire la réussite d'une première année universitaire ? Revue française de pédagogie, 119, 93-102.

Vygotski, L.S. (1985). Pensée et Langage. Paris : La Dispute.

Yarbro J., Arfstrom, K., McKnight K., McKnight P. (2014). Extension of a review of Flipped Learning. Repéré à http://flippedlearning.org/wpcontent/uploads/2016/07/Extension-of-FLipped-Learning-LIt-Review-June2014.pdf

Biographie de l'auteure

Anne Rohr est Maître de Conférence au Département Sciences du langage à l'Université Toulouse2 - Jean-Jaurès. Psycholinguiste de formation, elle travaille sur l'apprentissage de la langue écrite en contexte scolaire et universitaire. Elle est également responsable, pour les enseignements du français, de la Discipline Associé «Vers le professorat des écoles » proposée à l'Université Toulouse2 - Jean-Jaurès. 